TRANSACTIONS OF THE

AMERICAN MATHEMATTICAL SOCIETY

Volume 188, Issue 2, 1974

\title{
REPRESENTATION OF FUNCTIONS AS LIMITS OF MARTINGALES(1)
}

BY

\section{CHARLES W. LAMB}

ABSTRACT. In this paper we show that if $(\Omega, \mathcal{Y}, P)$ is a probability space and if $\left\{\mathcal{F}_{n}\right\}_{n \geq 1}$ is an increasing sequence of sub- $\sigma$-fields of $\mathcal{F}$ which satisfy an additional condition, then every real valued, $\mathcal{F}_{\infty}$-measurable function $f$ can be written as the a.e. limit of a martingale $\left\{f_{n}, \mathcal{F}_{n}\right\}_{n \geq 1}$. The case where $f$ takes values in the extended real line is also studied. A construction is given of a "universal" martingale $\left\{f_{n}, \Phi_{n}\right\}_{n \geq 1}$ such that any $\Phi_{\infty}$-measurable function is the a.e. limit of a suitably chosen subsequence.

1. Introduction. Throughout this paper $\left\{\mathcal{F}_{n}\right\}_{n \geq 1}$ will denote an increasing sequence of $\sigma$-fields on a probability space $(\Omega, \mathcal{F}, P)$. Let $\mathcal{F}_{\infty}$ be the smallest offield containing every $\mathfrak{F}_{n}$. Assume $\mathfrak{F}_{\infty} \subset \mathcal{F}$. A $d$-sequence of $\sigma$-fields is defined in $\$ 2$. The terminology $d$-sequence is used to indicate that the sequence is "disintègrating". If every $\mathcal{F}_{n}$ is atomic, then $\left\{\mathcal{F}_{n}\right\}_{n \geq 1}$ is a $d$-sequence if and only if no set in $\bigcup_{n \geq 1} \mathcal{F}_{n}$ is an atom of $\mathcal{F}_{\infty}$.

In $\$ 3$ we prove (Theorem 3.2) that if $\left\{\mathcal{F}_{n}\right\}_{n \geq 1}$ is a $d$-sequence and $f: \Omega \rightarrow$ $(-\infty, \infty)$ is $\mathfrak{F}_{\infty}$-measurable, then there exists a martingale $\left\{f_{n}, \mathcal{F}_{n}\right\}_{n \geq 1}$ such that $\lim _{n} f_{n}=f$ a.e. If $f$ is $P$-integrable, then we may take $f_{n}=E\left\{f \mid \mathcal{F}_{n}\right\}$ and the assumption that $\left\{\mathcal{F}_{n}\right\}_{n \geq 1}$ is a $d$-sequence is superfluous. The case of interest here is when $f$ is not $P$-integrable. The case where $\mathcal{F}_{n}=\mathcal{F}_{1}$ for all $n \geq 1$ shows that if $f$ is not $P$-integrable, then some additional assumptions are necessary. The above representation theorem was motivated by the work of R. F. Gundy [5] on orthogonal series and martingales and we obtain some of Gundy's results as special cases. Using our representation theorem, we prove a martingale version of a theorem of Marcinkiewicz [9] on the existence of "universal" antiderivatives (Theorem 3.3). $\$ 3$ concludes with a few comments on continuous parameter martingale representations and stochastic integrals.

In $\$ 4$ the case where $f$ has values in the extended real line $[-\infty, \infty]$ is considered. In [1] Chow has studied the concept of a regular sequence of atomic

Received by the editors December 26, 1972.

AMS (MOS) subject classifications (1970). Primary 60G45; Secondary 40A05.

Key words and phrases. Martingales, $H$-systems, uni versal martingales.

(1) Research supported in part by NRC Operating Grant $\mathbf{A 8 4 9 9 .}$ 
$\sigma$-fields. We show how to define the subset $\Omega_{r} \subset \Omega$ on which an arbitrary increasing sequence of $\sigma$-fields is regular. It turns out (Theorem 4.4) that if $\left\{\mathcal{F}_{n}\right\}_{n \geq 1}$ is a $d$-sequence, then an $\mathfrak{F}_{\infty}$-measurable function $f: \Omega \rightarrow[-\infty, \infty]$ can be written as the almost every where limit of a martingale $\left\{f_{n}, \mathcal{F}_{n}\right\}_{n \geq 1}$ if and only if $f$ is finite a.e. on $\Omega_{\text {r }}$

In this paper the notation $A^{\prime}$ is used for the complement of a set $A$ and $1[A]$ for the indicator function of $A$. The reader is referred to [3] or [10] for background material on martingales.

2. Measure-theoretic preliminaries. In order to define a $d$-sequence of $\sigma$ fields, we first prove a result on the existence of certain maximal $\delta$-splitting sets.

Proposition 2.1. Let $\mathcal{G} \subset \mathcal{H} \subset \mathcal{F}$ and $0<\delta \leq 1 / 2$. For $B \in \mathcal{H}$, let $S(B, \delta)=$ $\{0<P\{B \mid \mathcal{G}\} \leq \delta\}$. There exists a set $A \in \mathcal{H}$ such that

(i) $P\{A \mid \mathcal{G}\} \leq \delta$ a.e.,

(ii) $S(B, \delta) \subset S(A, \delta)$ a.e. for every $B \in \mathcal{H}$.

The set $S(A, \delta)$ is uniquely determined up to equivalence and $S(A, \delta) \in \mathcal{G}$.

Proof. Let $0<\delta \leq 1 / 2$ be fixed. We will write $S(B)$ for $S(B, \delta)$ in the remainder of the proof. For $B \in \mathcal{H}$, it is convenient to let $B^{*}=B \cap S(B)$. Note that $S(B)=S\left(B^{*}\right),\left(B^{*}\right)^{*}=B^{*}$, and $P\left\{B^{*} \mid \varrho\right\} \leq \delta$.

If $B_{i} \in \mathcal{H}$ and $B_{i}=B_{i}^{*}$ for $i=1$ and 2 , then $B_{3}=B_{1} \cup\left(B_{2} \cap S\left(B_{1}\right)^{\prime}\right)$ satisfies

(a) $B_{3} \in \mathcal{H}$,

(b) $B_{1} \subset B_{3}$,

(c)

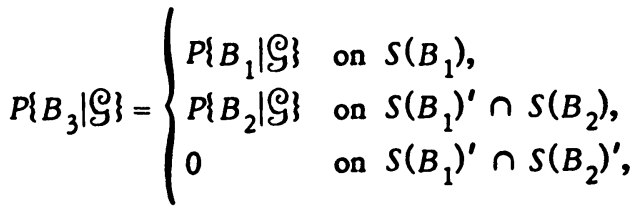

(d) $S\left(B_{1}\right) \cup S\left(B_{2}\right)=S\left(B_{3}\right)$,

(e) $B_{3}=B_{3}^{*}$.

Properties (a) and (b) are obvious and (c) follows by a routine calculation; (d) and (e) are implied by (c).

Let $\mathcal{S}=\{S(B): B \in \mathcal{H}\}=\left\{S(B): B \in \mathcal{H}, B=B^{*}\right\}$. Property (d) implies that $\mathcal{S}$ is a directed set under inclusion. Let $a=\sup \{P(S): S \in \delta\}$. An induction argument using properties (a) through (e) implies that there is an increasing sequence of sets $\left\{A_{n}\right\}_{n \geq 1}$ in $\mathcal{H}$ with $A_{n}=A_{n}^{*}$ and $\lim _{n} P\left\{S\left(A_{n}\right)\right\}=a$. The set $A=\bigcup_{n \geq 1} A_{n}$ satisfies $P\{A \mid \mathcal{G}\} \leq \delta$ and $P\{S(A)\}=a$. Therefore $S(A)$ is the unique maximal element of $\delta$, and the proof is complete. 
Definition 2.2. The set $S(A, \delta)$ of Proposition 2.1 is called the $\delta$-splitting set of $\mathcal{G}$ with respect to $\mathcal{H}$ and we write $S(A, \delta)=S(\delta)$. In addition, $S(1 / 2)$ is called the splitting set of $\varrho_{\text {with }}$ respect to $\mathcal{H}$ and we write $S(1 / 2)=S$.

This terminology is justified by the fact that if $B \in \mathcal{H}$ and

$$
\hat{B}=[B \cap\{0<P\{B \mid \varrho\} \leq 1 / 2\}] \cup\left[B^{\prime} \cap\{1 / 2<P\{B \mid \varrho\}<1\}\right],
$$

then $\{0<P\{B \mid \mathcal{G}\}<1\}=\{0<P\{\hat{B} \mid \mathcal{G}\} \leq 1 / 2\} \subset S$. If $\mathcal{H}$ is atomic, then $S$ is simply the union of all atoms of $@$ which split into two or more atoms of $\mathcal{H}$. More generally, if $\mathcal{H}$ is countably generated and $(\omega, B) \rightarrow p(\omega, B)$ is a regular version of the conditional probability of $\mathcal{H}$ given $\mathcal{G}$ (see [6]), then the complement of $S$ is equivalent to $\left\{\omega: p(\omega, B)=1\right.$ if $\omega \in B,=0$ if $\omega \in B^{\prime}$ for every $\left.B \in \mathcal{H}\right\}$. No use will be made of this last fact and its verification is left to the reader.

Definition 2.3. For $1 \leq m<n<\infty$, let $S(m, n)$ be the splitting set of $\mathcal{F}_{m}$ with respect to $\mathcal{F}_{n}$. If $\bigcup_{n: n>m} S(m, n)=\Omega$ a.e. for every $m \geq 1$, then $\left\{\mathcal{F}_{n}\right\}_{n \geq 1}$ is called a $d$-sequence.

Since $S(m, n) \subset S(m, n+1)$ for $1 \leq m<n<\infty,\left\{\mathcal{F}_{n}\right\}_{n \geq 1}$ is a $d$-sequence if and only if $\lim _{n} P\{S(m, n)\}=1$ for every $m \geq 1$. A sequence of atomic $\sigma$-fields $\left\{\mathcal{F}_{n}\right\}_{n \geq 1}$ is a $d$-sequence if and only if no set in $\bigcup_{n \geq 1} \mathfrak{F}_{n}$ is an atom of $\mathfrak{F}_{\infty}$.

Proposition 2.4. For $1 \leq m<n<\infty$, let $S(m, n, \delta)$ be the $\delta$-splitting set of $\mathcal{F}_{m}$ with respect to $\mathcal{F}_{n}$. If $\left\{\bar{F}_{n}\right\}_{n \geq 1}$ is a d-sequence, then $\bigcup_{n: n \geq m} S(m, n, \delta)=\Omega$ a.e. for every $m \geq 1$ and $0<\delta \leq 1 / 2$.

Proof. It suffices to prove the proposition when $\delta=(1 / 2)^{i}$ for $i \geq 1$. The case $i=1$ follows from the definition of a $d$-sequence. Assume the result is true for $\delta=(1 / 2)^{k}$. Let $\epsilon>0$ and $m \geq 1$ be given. Choose an integer $n>m$ and a set $A_{0} \in \mathcal{F}_{n}$ such that $P\left\{A_{0} \mid \mathcal{F}_{m}\right\} \leq 1 / 2$ and $P\left\{0<P\left\{A_{0} \mid \mathcal{F}_{m}\right\} \leq 1 / 2\right\}>1-\epsilon$. Using the induction hypothesis and arguing as in the proof of Proposition 2.1, we choose an increasing sequence of sets $\left\{A_{j}\right\}_{j \geq 1}$ such that $A_{j} \in \mathcal{F}_{n+j}, P\left\{A_{j} \mid \mathcal{F}_{n}\right\} \leq(1 / 2)^{k}$, and

We have

$$
\lim _{j} P\left\{0<P\left\{A_{j} \mid F_{n}\right\} \leq(1 / 2)^{k}\right\}=1 \text {. }
$$

$$
\begin{aligned}
P\left\{A_{0} \cap A_{j} \mid \mathcal{F}_{m}\right\} & =E\left\{P\left\{A_{0} \cap A_{j} \mid \mathcal{F}_{n}\right\} \mid \mathcal{F}_{m}\right\}=E\left\{1\left[A_{0}\right] P\left\{A_{j} \mid \mathcal{F}_{n}\right\} \mid \mathcal{F}_{m}\right\} \\
& \leq(1 / 2)^{k} E\left\{1\left[A_{0}\right] \mid \mathcal{F}_{m}\right\} \leq(1 / 2)^{k+1} .
\end{aligned}
$$

The set where $P\left\{A_{0} \cap A_{j} \mid \mathcal{F}_{m}\right\}>0$ increases to the set where

$$
E\left\{1\left[A_{0}\right] \lim _{j} P\left\{A_{j} \mid \mathfrak{F}_{n}\right\} \mid \mathfrak{F}_{m}\right\}>0 \text {, }
$$


and this latter set is equivalent to the set where $E\left\{1\left[A_{0}\right] \mid \mathcal{F}_{m}\right\}>0$. Hence

$$
\begin{aligned}
& \lim _{j} P\left\{S\left(m, n+j,(1 / 2)^{k+1}\right)\right\} \geq \lim _{j} P\left\{0<P\left\{A_{0} \cap A_{j} \mid \mathcal{F}_{m}\right\} \leq(1 / 2)^{k+1}\right\} \\
& =P\left\{0<P\left\{A_{0} \mid \mathcal{F}_{m}\right\} \leq(1 / 2)\right\}>1-\epsilon \text {, }
\end{aligned}
$$

and the proof is complete.

3. Representation of real valued functions.

Proposition 3.1. Let $\left\{\mathcal{F}_{n}\right\}_{n \geq 1}$ be a d-sequence. Let $f: \Omega \rightarrow(-\infty, \infty)$ be $\mathcal{F}_{\infty}$-measurable and $P$-integrable. If $n_{0} \geq 1$ is an integer, then there exists a martingale $\left\{f_{n}, \mathcal{F}_{n}\right\}_{n \geq 1}$ sucb that

(i) $f_{n}=0$ a.e. for $1 \leq n \leq n_{0}$,

(ii) $\lim _{n} f_{n}=f$ a.e.

If, in addition, $|f| \leq M$ a.e., then there exists a sequence of constants $\left\{M_{n}\right\}_{n \geq 1}$ such that

(iii) $\left|f_{n}\right| \leq M_{n}$ a.e. for every $n \geq 1$.

Proof. We first construct a martingale $\left\{g_{n}, \mathcal{F}_{n}\right\}_{n \geq 1}$ such that $g_{n}=E\left\{f \mid \mathcal{F}_{n}\right\}$ for $1 \leq n \leq n_{0}$, and $\lim _{n \rightarrow \infty} g_{n}=0$ a.e. Once this is done, the martingale $\left\{f_{n}, \mathcal{F}_{n}\right\}_{n \geq 1}$ is defined by $f_{n}=E\left\{f \mid \mathcal{F}_{n}\right\}-g_{n}$.

Choose $\left\{\delta_{k}\right\}_{k \geq 1}$ such that $\delta_{k}>0$ and $\Sigma_{k \geq 1} \delta_{k}<\infty$. Using Proposition 2.3, we may choose $\left\{m_{k}, A_{k}, S_{k}\right\}_{k \geq 0}$ by induction such that $m_{0}=n_{0}, m_{k}<m_{k+1}, S_{k}$ is the $\delta_{k}$-splitting set of $\mathcal{F}_{m_{k}}$ with respect to $\mathcal{F}_{m_{k+1}}, A_{k} \in \mathcal{F}_{m_{k+1}}$ corresponds to $S_{k}$ as in Proposition 2.1, and

$$
P\left\{S_{k}\right\}>1-\delta_{k}
$$

Define a sequence of functions $\left\{g_{m_{k}}\right\}_{k \geq 1}$ by induction as follows:

$$
g_{m_{0}}=E\left\{f \mid \mathcal{F}_{m_{0}}\right\}, \quad g_{m_{k+1}}= \begin{cases}g_{m_{k}} 1\left[A_{k}\right] / P\left\{A_{k} \mid \mathcal{F}_{m_{k}}\right\} & \text { on } S_{k}, \\ g_{m_{k}} & \text { on } S_{k}^{\prime} .\end{cases}
$$

An induction argument shows that $E\left\{\left.\right|_{m_{k}} \mid\right\}=E\left\{\left.\right|_{m_{0}} \mid\right\} \leq E\{|f|\}<\infty$, and a direct calculation yields $E\left\{g_{m_{k+1}} \mid \mathcal{F}_{m_{k}}\right\}=g_{m_{k}}$. For $m_{k}<n<m_{k+1}$, let $g_{n}=$ $E\left\{g_{m_{k+1}} \mid \mathcal{F}_{n}\right\}$. It follows that $\left\{g_{n}\right\}_{n \geq 1}$ is an $L^{1}$-bounded martingale and $g_{n}=$ $E\left\{f \mid \mathcal{F}_{n}\right\}$ for $1 \leq n \leq m_{0}=n_{0}$.

We now show that $g_{n}$ is eventually equal to 0 a.e. It suffices to show that $g_{m_{k}}$ is eventually equal to 0 , since $g_{m_{k}+1}=\cdots=g_{m_{k}+1}=0$ on the set where $\boldsymbol{g}_{m_{k}}=0$. Equation (3.1) and the Borel-Cantelli lemma imply that 


$$
P\left\{\underset{k}{\lim \sup _{k}} S_{k}^{\prime}\right\}=0
$$

According to (3.3) and (3.2), it suffices to show that $P\left\{\lim \sup _{k} A_{k}\right\}=0$, and this follows from $P\left\{A_{k}\right\}=E\left\{P\left\{A_{k} \mid \mathcal{F}_{m_{k}}\right\}\right\} \leq \delta_{k}$. Parts (i) and (ii) are now proved.

To prove (iii), we need only replace $A_{k}$ by $\hat{A}_{k}=A_{k} \cap\left\{P\left\{A_{k} \mid \mathcal{F}_{m_{k}}\right\} \geq \epsilon_{k}\right\}$ where $\epsilon_{k}>0$ is chosen small enough such that $S_{k}=\left\{0<P\left\{\hat{A}_{k} \mid \mathscr{F}_{m_{k}}\right\} \leq \delta_{k}\right\}$ satisfies (3.1). It follows that $P\left\{\hat{A}_{k} \mid \mathcal{F}_{m_{k}}\right\} \geq \epsilon_{k}$ on $S_{k}$ and (3.2) implies that $g_{m_{k}}$ is bounded for every $k \geq 1$. The proof is now complete.

We will say that the martingale $\left\{f_{n}, \mathcal{F}_{n}\right\}_{n \geq 1}$ of Proposition 3.1 is associated with $n_{0}$ and $f$.

Theorem 3.2. Let $\left\{\mathcal{F}_{n}\right\}_{n \geq 1}$ be a d-sequence. Let $f: \Omega \rightarrow(-\infty, \infty)$ be $\mathcal{F}_{\infty}$ measurable. There exists a martingale $\left\{f_{n}, \mathcal{F}_{n}\right\}_{n \geq 1}$ and a sequence of constants $\left\{M_{n}\right\}_{n \geq 1}$ such that

(i) $\left|f_{n}\right| \leq M_{n}$ a.e. for every $n \geq 1$,

(ii) $\lim _{n} f_{n}=f$ a.e.

Proof. It suffices to assume that $f \geq 0$. Let $B_{i}=\{i-1 \leq f<i\}$ for $i \geq 1$. Choose $\left\{\epsilon_{i j}\right\}_{i, j \geq 1}$ such that $\epsilon_{i j}>0$ and $\Sigma_{i, j \geq 1} \epsilon_{i j}<\infty$. Choose $\left\{m_{i j}, C_{i j}\right\}_{i, j \geq 1}$ such that $C_{i j} \in \mathcal{F}_{m_{i j}}$, and if $D_{i j}=\bigcup_{k=1}^{j} C_{i k}$, then

$$
P\left\{\left(B_{i} \cap D_{i(j-1)}^{\prime}\right) \Delta C_{i j}\right\}<\epsilon_{i j}
$$

for $i, j \geq 1$. We may assume that $\left\{C_{i j}\right\}_{j \geq 1}$ is a disjoint sequence of sets for every $i \geq 1$, and that

$$
\lim _{i, j} m_{i j}=\infty \text {. }
$$

Form a martingale $\left\{f_{n}^{i j}, \mathcal{F}_{n}\right\}_{n \geq 1}$ as in Proposition 3.1 which is associated with $m_{i j}$ and $f 1\left[B_{i} \cap C_{i j}\right]$. It follows from the construction of $\left\{f^{i j}, \mathcal{F}_{n}\right\}_{n \geq 1}$ that

$$
f_{n}^{i j}=0 \text { on } C_{i j}^{\prime} \text { for every } n \geq 1 \text {. }
$$

Note also that $\lim _{n} f_{n}^{i j}=f$ on $B_{i} \cap C_{i j}$, and $\lim _{n} f_{n}^{i j}=0$ otherwise.

We claim that

(a) $P\left\{\bigcup_{i, j \geq 1} C_{i j}\right\}=1$,

(b) $P\left\{\lim \sup _{i, j} C_{i j}\right\}=0$.

Property (a) follows from $B_{i} \subset \bigcup_{j \geq 1} C_{i j}$ a.e. Property (b) follows from (3.4) and

$$
\underset{i, j}{\lim \sup } C_{i j} C \lim \sup _{i, j}\left(B_{i} \cap D_{i(j-1)}\right) \Delta C_{i j}
$$


Now consider the sum

$$
f_{n}=\sum_{i, j \geq 1} f_{n}^{i j}
$$

Equation (3.5) and the fact that $f_{n}^{i j}=0$ for $1 \leq n \leq m_{i j}$ imply that $\left\{f_{n}, \mathcal{F}_{n}\right\}_{n \geq 1}$ is a well-defined martingale which satisfies (i). Properties (a), (b) and (3.6) inply that (ii) holds and the proof is complete.

The motivation for Theorem 3.2 came from the work of $R, F$. Gundy [s] on orthogonal series and martingales. In fact, the proof of Theorem 3.2 was modelled after Gundy's proof that any Borel measurable function $f:[0,1] \rightarrow(-\infty, \infty)$ can be written as the a.e. (Lebesgue) limit of an orthogonal series $\Sigma_{k \geq 1} c_{k} \phi_{k}$, where $\left\{\phi_{k}\right\}_{k \geq 1}$ is a complete $H$-system. Recall that an orthonormal system of functions $\left\{\phi_{k}\right\}_{k \geq 1}$ is an $H$-system if, for every square integrable function $f$ on $[0,1]$,

$$
E\left\{f \mid \phi_{1}, \cdots, \phi_{n}\right\}=\sum_{k \geq 1}\left(f, \phi_{k}\right) \phi_{k},
$$

where $\left(f, \phi_{k}\right)=\int_{0}^{1} f \phi_{k} d x$. Equivalently, an orthonormal set $\left\{\phi_{k}\right\}_{k \geq 1}$ is an $H$ system if $\mathcal{F}\left(\phi_{1}, \cdots, \phi_{n}\right)$ is atomic with exactly $n$-atoms for $n \geq 1$. It is easy to see that any martingale $\left\{f_{n}, \mathcal{F}\left(\phi_{1}, \ldots, \phi_{n}\right)\right\}_{n \geq 1}$ can be written as $f_{n}=\Sigma_{k \geq 1} c_{k} \phi_{k}$ for suitable constants $\left\{c_{k}\right\}_{k \geq 1}$, and hence Gundy's result follows from Theorem 3.2.

Gundy's treatment was closely related to a classical differentiation theorem of Lusin (see [11, p. 217]) which states that for a Borel measurable function $f:$ $[0,1] \rightarrow(-\infty, \infty)$, there exists a continuous function $F:[0,1] \rightarrow(-\infty, \infty)$ such that $F^{\prime}=f$ a.e. (Lebesgue). Theorem 3.2 may be viewed as a martingale generalization of Lusin's theorem. Marcinkiewicz [9] used Lusin's theorem to prove the existence of certain "universal" functions $F$ with the property that for every Borel measurable function $f:[0,1] \rightarrow[-\infty, \infty]$, there exists a subsequence $\left\{b_{n}\right\}_{n \geq 1}$ of strictly positive numbers decreasing to 0 such that

$$
\lim _{n}\left[F\left(x+b_{n}\right)-F(x)\right] / h_{n}=f \text { a.e. }
$$

We now prove a martingale version of this result.

Theorem 3.3. Let $\left\{\mathcal{F}_{n}\right\}_{n \geq 1}$ be a d-sequence and assume that $\mathfrak{F}_{\infty}$ is countably generated. There exists a martingale $\left\{f_{n}, \mathcal{F}_{n}\right\}_{n \geq 1}$ with the following universal convergence property: If $f: \Omega \rightarrow[-\infty, \infty]$ is $\mathfrak{F}_{\infty}$-measurable, then there exists a subsequence $\left\{n_{k}\right\}_{k \geq 1}$ of the positive integers sucb that $\lim _{k} f_{n_{k}}=f$ a.e.

Proof. There exists a countable set of real valued, $\mathcal{F}_{\infty}$-measurable functions $\left\{b_{n}\right\}_{n \geq 1}$ such that every $f$ as described above can be written as the a.e. limit of a suitable subsequence $\left\{b_{n_{k}}\right\}_{k \geq 1}$. Choose $\left\{\delta_{k}\right\}_{k \geq 1}$ such that $\delta_{k}>0$ and $\Sigma_{k \geq 1} \delta_{k}<\infty$. 
Define $\left\{m_{k},\left\{f_{n}^{k}, \mathcal{F}_{n}\right\}_{n \geq 1}\right\}_{k \geq 0}$ by induction as follows: Let $m_{0}=0, f_{n}^{0}=0$ for every $n \geq 1$. Assuming that $\left\{m_{i},\left\{f_{n}^{i}, \mathcal{F}_{n}\right\}_{n \geq 1}\right\}_{1 \leq i \leq t}$ have been defined, we choose $m_{k+1}$ such that

$$
P\left\{\left|b_{k}-\sum_{i=1}^{k} f_{n}^{i}\right| \geq \delta_{k}\right\}<\delta_{k}
$$

if $n \geq m_{k}$. Let $\left\{f_{n}^{k+1}, \mathcal{F}_{n}\right\}_{n \geq 1}$ be a martingale such that $f_{n}^{k+1}=0$ if $1 \leq n \leq$ $m_{k+1}$, and $\lim _{n} f_{n}^{k+1}=b_{k+1}-b_{k}$ a.e. Such a martingale exists by Theorem 3.2. Now let $f_{n}=\Sigma_{k \geq 1} f_{n}^{k}$. It follows that $\left\{f_{n}, \mathcal{F}_{n}\right\}_{n \geq 1}$ is a well-defined martingale and

$$
P\left\{\left|f_{m_{k+1}}-b_{k}\right| \geq \delta_{k}\right\}<\delta_{k}
$$

If $f$ is the a.e. limit of $\left\{b_{n_{k}}\right\}_{k \geq 1}$, then (3.7) and the Borel-Cantelli lemma imply that the subsequence of $\left\{f_{n}, \mathfrak{F}_{n}\right\}_{n \geq 1}$ corresponding to $\left\{m_{n_{k+1}}\right\}_{k \geq 1}$ converges to $f$ a.e., and the proof is complete.

We will call a martingale with the property described in Theorem 3.4 a universal martingale. By taking subsequences of universal martingales we may construct various examples of strangely behaved martingales. For example, Luis Baez-Duarte [8] gave an example of a martingale which converges in measure but not a.e. To obtain a large class of such examples it suffices to take (in the proof of Theorem 3.3) $\left\{b_{n_{k}}\right\}_{k \geq 1}$ converging in measure but not a.e. A similar comment applies to convergence in distribution (see [4]).

Let $\left\{\mathcal{F}_{t}\right\}_{t \geq 0}$ be a family of $\sigma$-fields on a probability space $(\Omega, \mathcal{F}, P)$. We will say that $\left\{\mathcal{F}_{t}\right\}_{t \geq 0}$ is a d-family if $\left\{\mathcal{F}_{t_{n}}\right\}_{n \geq 1}$ is a $d$-sequence for some (and hence every) increasing sequence $\left\{t_{n}\right\}_{n \geq 1}$ with $\lim _{n} t_{n}=\infty$. It is easy to adapt the results of this section to the continuous case and we obtain the following result:

Theorem 3.4. Let $\left\{\mathcal{F}_{t}\right\}_{t \geq 0}$ be a d-family. Let $f: \Omega \rightarrow(-\infty, \infty)$ be $\mathcal{F}_{\infty}$-measurable. There exists a martingale $\left\{f_{t}, \mathcal{F}_{t}\right\}_{t \geq 0}$ and an increasing function $M:[0, \infty)$ $\rightarrow[0, \infty)$ such that

(i) $\left|f_{t}\right| \leq M_{t} a_{0} e_{0}$ for every $t \geq 0$.

(ii) $\lim _{t \in Q} f_{t}=f$ a.e., where $Q$ is any countably dense subset of $[0, \infty)$.

Let $\left(\Omega, \mathcal{F}, \mathcal{F}_{t}, X_{t}, \mathcal{O}_{t}, P^{x}\right)$ be a Hunt process with a locally compact and countably generated state space (see [2]). If there are no absorbing points in the state space, then $\left\{\mathcal{F}_{t}\right\}_{t \geq 0}$ is easily shown to be a $d$-family with respect to 
each measure $P^{\mu}$. It follows from Theorem 3.4 that if a measure $P^{\mu}$ is given and $f: \Omega \rightarrow(-\infty, \infty)$ is $\mathfrak{F}_{\infty}$-measurable, then there is a separable martingale $\left\{f_{t}, \mathcal{F}_{t}\right\}_{t \geq 0}$ such that $\lim _{t} f_{t}=f$ a.e. For example, if we consider 1 -dimensional Brownian motion with initial distribution $\mu$, and if $\left\{f_{t}, \mathcal{F}_{t}\right\}_{t \geq 0}$ is chosen to be square integrable, then by a theorem of Kunita and Watanabe [6]

$$
f_{t}=\int_{0}^{t} \psi_{s} d X_{s}
$$

for some nonanticipating functional $\left\{\psi_{s}\right\}_{s \geq 0}$. Roughly speaking, this means that Brownian motion can be controlled to converge to any real valued measurable function $f$.

4. Representation of extended real valued functions. If $f: \Omega \rightarrow[-\infty, \infty]$ is $\mathcal{F}_{\infty}$-measurable and $\left\{\mathcal{F}_{n}\right\}_{n \geq 1}$ is a $d$-sequence of $\sigma$-fields, then it does not necessarily follow that there is a martingale $\left\{f_{n}, \mathcal{F}_{n}\right\}_{n \geq 1}$ which converges to $f$ a.e. Chow [1] proved that if $\left\{\mathcal{F}_{n}\right\}_{n \geq 1}$ is a regular sequence of atomic $\sigma$-fields, then no martingale $\left\{f_{n}, \mathcal{F}_{n}\right\}_{n \geq 1}$ can converge to $\infty$ (or $-\infty$ ) on a set of strictly positive $P$-measure.

Definition 4.1. For $n \geq-1$, let $S_{n}(\delta)$ be the $\delta$-splitting set of $\mathcal{F}_{n}$ with respect to $\mathcal{F}_{n+1}$. Let $\left\{\delta_{k}\right\}_{k \geq 1}$ be a sequence of strictly positive numbers decreasing to 0 . The regular part $\Omega_{r}$ of $\Omega$ with respect to $\left\{\mathcal{F}_{n}\right\}_{n \geq 1}$ is defined as the complement of the set

$$
\bigcap_{k \geq 1} \lim \sup _{n} S_{n}\left(\delta_{k}\right)
$$

The sets $\lim \sup _{n} S_{n}(\delta)$ decrease as $\delta$ decreases and hence the intersection in (4.1) is independent of the particular sequence $\left\{\delta_{k}\right\}_{k \geq 1}$. If $\left\{\mathcal{F}_{n}\right\}_{n \geq 1}$ is a sequence of atomic $\sigma$-fields, then $\left\{\mathcal{F}_{n}\right\}_{n \geq 1}$ is regular in Chow's sense if and only if there is a $\delta_{k}$ such that $S_{n}\left(\delta_{k}\right)=\varnothing$ for all $n \geq 1$. We sharpen Chow's convergence result as follows:

Theorem 4.2. If $\left\{f_{n}, \mathcal{F}_{n}\right\}_{n \geq 1}$ is a martingale, then $\lim _{n} f_{n}$ exists and is finite a.e. on the set

$$
\Omega_{r} \cap\left(\left\{\sup _{n} f_{n}<\infty\right\} \cup\left\{\inf _{n} f_{n}>-\infty\right\}\right) .
$$

Proof. Let $m \geq 1$ be an integer and $\lambda \epsilon(-\infty, \infty)$ be given. Define a stopping time with respect to $\left\{\mathcal{F}_{n}\right\}_{n \geq 1}$ by

$$
T(m, \lambda)=\inf \left\{i \geq m: P\left\{f_{i+1}>\lambda \mid F_{i}\right\}>0\right\} \text {. }
$$


It follows that $\left\{f_{T(m, \lambda) \wedge n}, \mathcal{F}_{n}\right\}_{n \geq 1}$ is a martingale with $f_{T(m, \lambda) \wedge_{n}} \leq f_{m} \vee \lambda$ for $n \geq m$. Hence $\lim _{n} f_{T(m, \lambda) \wedge_{n}}$ exists and is finite a.e. To prove our theorem, it suffices to show that for a.e. $\omega_{0} \in \Omega_{r} \cap\left\{\sup _{n} f_{n}<+\infty\right\}$ there is a corresponding pair $(m, \lambda)$ such that $T(m, \lambda)\left(\omega_{0}\right)=\infty$. We omit references to exceptional sets of $P$-measure 0 in the arguments which follow.

If $\omega_{0} \in \Omega_{r} \cap\left\{\sup _{n} f_{n}<\infty\right\}$, then

(a) there is a number $\delta>0$ and an integer $j \geq 1$ such that $\omega_{0} \in S_{n}(\delta)^{\prime}$ for $n \geq j$.

(b) there is a number $\lambda \in(-\infty, \infty)$ such that $\sup _{n} f_{n}\left(\omega_{0}\right) \leq \lambda$. It follows from (a) that

$$
P\left\{f_{i+1}>\lambda \mid \mathcal{F}_{i}\right\}\left(\omega_{0}\right)>\delta \text { or }=0 \text { for } i \geq j
$$

Now

$$
\begin{aligned}
0 & \leq P\left\{f_{i+1}>\lambda \mid F_{i}\right\}\left(\omega_{0}\right) \leq P\left\{\sup _{n} f_{n}>\lambda \mid F_{i}\right\}\left(\omega_{0}\right) \\
& \rightarrow\left\{\begin{array}{c}
1 \text { if } \sup _{n} f_{n}\left(\omega_{0}\right)>\lambda, \\
0 \text { if } \sup _{n} f_{n}\left(\omega_{0}\right) \leq \lambda,
\end{array}\right.
\end{aligned}
$$

as $i \rightarrow \infty$. It follows from (4.3) and (4.4) that there is an integer $m \geq j$ such that $P\left\{f_{i+1}>\lambda \mid F_{i}\right\}\left(\omega_{0}\right)=0$ for $i \geq m$. Hence $T(m, \lambda)\left(\omega_{0}\right)=\infty$, and the proof is complete.

Theorem 4.3. If $B \in \mathcal{F}_{\infty}$ and $B \subset \Omega_{r}^{\prime}$ then there exists a martingale $\left\{f_{n}, \mathcal{F}_{n}\right\}_{n \geq 1}$ sucb that $\lim _{n} f_{n}$ exists and is finite a.e. on $B^{\prime}$, and $\lim _{n} f_{n}=\infty$ a.e. on $B$.

Proof. Choose $\left\{\delta_{k}\right\}_{k \geq 1}$ such that $\delta_{k}>0$ and $\Sigma_{k \geq 1} \delta_{k}<\infty$. Choose $\left\{m_{k}, B_{k}\right\}_{k \geq 1}$ such that $B_{k} \in \mathcal{F}_{m_{k}}$ and $P\left\{B \Delta B_{k}\right\}<\delta_{k}$. Define a sequence of stopping times $\left\{T_{k}\right\}_{k \geq 0}$ with respect to $\left\{\mathcal{F}_{n}\right\}_{n \geq 1}$ by induction as follows:

$$
T_{0}=1, \quad T_{k+1}=\inf \left\{i>T_{k} \vee m_{k}: \omega \in S_{i}\left(\delta_{k+1}\right)\right\},
$$

where $S_{i}\left(\delta_{k}\right)$ is the $\delta_{k}$-splitting set of $\mathcal{F}_{i}$ with respect to $\mathfrak{F}_{i+1}$. Let $A_{i}\left(\delta_{k}\right) \epsilon$ $\mathcal{F}_{i+1}$ correspond to $S_{i}\left(\delta_{k}\right)$ as in Proposition 2.1. Note that $T_{k}<T_{k+1}<\infty$ on $B$ for $k \geq 1$, and $T_{i}$ is eventually equal to $\infty$ on $B^{\prime}$.

For $k \geq 1$, let

$$
A_{T_{k}}=\bigcup_{i \geq 1} A_{i}\left(\delta_{k}\right) \cap\left\{T_{k}=i\right\} \in \mathcal{F}_{T_{k}+1}, \quad S_{T_{k}}=\bigcup_{i \geq 1} S_{i}\left(\delta_{k}\right) \cap\left\{T_{k}=i\right\} \in \mathcal{F}_{T_{k}} \text {, }
$$

and note that 


$$
\begin{gathered}
S_{T_{k}}=\left\{T_{k}<\infty\right\}, \\
P\left\{A_{T_{k}} \mid \mathcal{F}_{T_{k}}\right\}=P\left\{A_{i}\left(\delta_{k}\right) \mid \mathcal{F}_{i}\right\} \text { a.e. on }\left\{T_{k}=i\right\} .
\end{gathered}
$$

Equations (4.5) and (4.6) imply that

$$
P\left\{A_{T_{k}} \mid \mathcal{F}_{T_{k}}\right\} \leq \delta_{k} \quad \text { a.e., } \quad\left\{0<P\left\{A_{T_{k}} \mid \mathcal{F}_{T_{k}}\right\} \leq \delta_{k}\right\}=S_{T_{k}} \cdot
$$

Define a sequence of functions $\left\{f_{n}\right\}_{n \geq 1}$ by induction as follows:

$$
\begin{gathered}
f_{1}=\cdots=f_{T_{1}}=0, \\
f_{T_{k}+1}=\cdots=f_{T_{k+1}}= \begin{cases}f_{T_{k}+1} & \text { on } A_{T_{k}}^{\prime} \cap B_{k}, \\
g_{k} & \text { on } A_{T_{k}} \cap B_{k}, \\
f_{T_{k}} & \text { on } B_{k}^{\prime},\end{cases}
\end{gathered}
$$

where

$$
g_{k}=\left(f_{T_{k}} P\left\{A_{T_{k}} \mid \mathcal{F}_{T_{k}}\right\}-P\left\{A_{T_{k}}^{\prime} \mid \mathcal{F}_{T_{k}}\right\}\right) P\left\{A_{T_{k}} \mid \mathcal{F}_{T_{k}}\right\}^{-1}
$$

on $A_{T_{k}} \cap B_{k^{\circ}}$. Routine calculations show that $f_{n}$ is $P$-integrable for every $n \geq 1$, and that $\left\{f_{n}, \mathcal{F}_{n}\right\}_{n \geq 1}$ is a martingale.

Now $P\left\{A_{T_{k}}\right\}=E\left\{P\left\{A_{T_{k}} \mid \mathcal{F}_{T_{k}}\right\}\right\} \leq \delta_{k}$, and hence $P\left\{\lim \sup _{k} A_{T_{k}}\right\}=0$. In addition, $P\left\{\lim \sup _{k}\left(B \Delta B_{k}\right)\right\}=0$. Hence, if $\omega_{0} \in B$, then $\omega_{0}$ is eventually in $A_{T_{k}}^{\prime} \cap B_{k}$ and $\lim _{n} f_{n}\left(\omega_{0}\right)=\infty$. On the other hand if $\omega_{0} \in B^{\prime}$, then $\omega_{0}$ is eventually in $B_{k}^{\prime}$ and $f_{n}\left(\omega_{0}\right)$ is eventually constant. The proof is complete.

If, in (4.7) we replace $f_{T_{k}}+1$ by $f_{T_{k}}+(-1)^{k}$, then the martingale $\left\{f_{n}, \mathcal{F}_{n}\right\}_{n \geq 1}$ will oscillate boundedly on $B$ and converge on $B^{\prime}$. Thus, either of the two types of behavior ruled out on $\Omega_{r}$ by Theorem 4.2 can occur on $\Omega_{r}^{\prime}$. The following theorem is the main result of this paper.

Theorem 4.4. Let $\left\{\mathcal{F}_{n}\right\}_{n \geq 1}$ be a d-sequence. Let $f: \Omega \rightarrow[-\infty, \infty]$ be $\mathcal{F}_{\infty}$ measurable. There exists a martingale $\left\{f_{n}, \mathcal{F}_{n}\right\}_{n \geq 1}$ sucb that $\lim _{n} f_{n}=f$ a.e. if and only if $|f|<\infty$ a.e. on $\Omega_{r}$.

Proof. The function $/$ must be finite a.e. on $\Omega_{r}$ by Theorem 4.2. For the converse, we may assume that $f \geq 0$. If $B=\{f=\infty\}$, then Theorem 4.3 shows that there is a martingale $\left\{b_{n}, \mathcal{F}_{n}\right\}_{n \geq 1}$ such that $\lim _{n} b_{n}$ exists and is finite a.e. on $B^{\prime}$ and $\lim _{n} b_{n}=\infty$ a.e. on $B$. Let $b=f-\lim _{n} b_{n}$ on $B^{\prime}$ and $b=0$ on $B$. 
According to Theorem 3.2, there exists a martingale $\left\{g_{n}, \mathcal{F}_{n}\right\}_{n \geq 1}$ such that $\lim _{n} g=b$ a.e. The proof is completed by taking $f_{n}=g_{n}+b_{n}$.

In order to find $\mathrm{H}$-systems of orthonormal functions which can be used to represent every extended real valued, Borel measurable function $f$ on $[0,1]$, Gundy [5] introduced the concept of an $H^{*}$-system. We will not repeat the definition of an $H^{*}$-system here, but remark that if $\left\{\phi_{k}\right\}_{k \geq 1}$ is an $H^{*}$-system, then $P\left\{\Omega_{r}\right\}=0$ and Gundy's representation theorem follows from Theorem 4.4.

\section{REFERENCES}

1. Y. S. Chow, Convergence theorems of martingales, Z. Wahrscheinlichkeitstheorie und Verw. Gebiete 1 (1962/63), 340-346. MR 27 \#807.

2. R. Blumenthal and R. Getoor, Markov processes and potential theory, Pure and Appl. Math., vol. 29, Academic Press, New York, 1968. MR 41 \#9348.

3. J. L. Doob, Stochastic processes, Wiley, New York; Chapman \& Hall, London, 1953. MR 15, 445.

4. D. Gilat, Convergence in distribution, convergence in probability and almost sure convergence of discrete martingales, Ann. Math. Statist. 43 (1972), 1374-1379.

5. R. F. Gundy, Martingale theory and pointwise convergence of certain orthogonal series, Trans. Amer. Math. Soc. 124 (1966), 228-248. MR 34 \# 4802.

6. H. Kunita and S. Watanabe, On square integrable martingales, Nagoy a Math. J. 30 (1967), 209-245. MR 36 \#945.

7. M. Loève, Probability theory. Foundations. Random sequences, 3rd ed., University Series in Higher Math., Van Nostrand, Princeton, N.J., $1963 . \quad$ MR 34 \#3596.

8. Luis Báez-Duarte, An a.e. divergent martingale that converges in probability, J. Math. Anal. Appl. 36 (1971), 149-150. MR 43 \#4096.

9. J. Marcinkiewicz, Sur les nombres dérivés, Fund. Math. 24 (1935), 305-308.

10. P. A. Meyer, Probability and potentials, Blaisdell, Waltham, Mass., 1966. MR 34 \# 5119.

11. S. Saks, Theory of the integral, 2nd rev. ed., Monografie Mat., vol. VI, PWN, Warsaw, 1937; reprint, Dover, New York, 1964. MR 29 \# 4850.

DEPARTMENT OF MATHEMATICS, UNIVERSITY OF BRITISH COLUMBIA, VANCOUVER, BRITISH COLUMBIA, CANADA 